\title{
Students' Perceptions of Team-Based Learning by Individual Characteristics in a Medical School
}

Kwi Hwa Park ${ }^{1}$, Chang-Hyu Choi ${ }^{2}$, Yang-Bin Jeon ${ }^{2}$, Kook-Yang Park ${ }^{2}$ and Chul-Hyun Park ${ }^{2}$

Departments of ${ }^{1}$ Medical Education and ${ }^{2}$ Cardiothoracic Surgery, Gachon University School of Medicine, Incheon, Korea

\section{일개 의과대학 학생들의 개인적 특성에 따른 팀바탕학습 인식도 조사}

가천대학교 의학전문대학원 ${ }^{1}$ 의학교육실, ${ }^{2}$ 흥부외과학교실

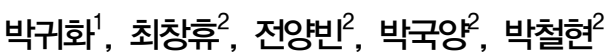

Purpose: The purpose of this study was to examine medical students' perceptions of team-based learning (TBL) according to their individual characteristics: gender, team efficacy, interpersonal understanding, proactivity in problem solving, and academic ability. Methods: Thirty-eight second-year medical students who took an integrated cardiology course participated in this study; 28 were male and 10 were female. A questionnaire on individual characteristics and a questionnaire on the perception of TBL were administered, and the scores of individual characteristics were grouped into three: high, middle, and low. The data were analyzed by t-test, analysis of variance, and multiple regression analysis.

Results: The TBL efficacy perception scale consisted of 3 factors: team skill, learning ability, and team learning. The group of male students and the group of students with high academic ability recognized the effect of TBL on improvements in learning ability more than females and those with low academic ability. The group of students with high team efficacy reported that TBL was effective with regard to team skill improvement. The group of students with high scores on interpersonal understanding and high proactive problem solving tended to perceive the TBL's effect on team skill improvement. Team efficacy and proactivity in problem solving had a positive effect on the perception of TBL.

Conclusion: Medical students' perceptions of the effectiveness of TBL differ according to individual characteristics. The results of this study suggest that these individual characteristics should be considered in planning of team learning, such as TBL, to have a positive impact and stronger effects.

Key Words: Team efficacy, Interpersonal understanding, Proactivity in problem solving, Academic ability, Team-based learning

Received: December 13, $2012 \bullet$ Revised: February 15, $2013 \bullet$ Accepted: February 25, 2013 Corresponding Author: Chul-Hyun Park

Department of Cardiothoracic Surgery, Gachon University School of Medicine, 38 Dokjeom-ro 3beon-gil, Namdong-gu, Incheon 405-835, Korea

Tel: +82.70.7120.7902 Fax:+82.32.464.4004 email: cdgpch@gilhospital.com
Korean J Med Educ 2013 Jun; 25(2): 113-122. http://dx.doi.org/10.3946/kjme.2013.25.2.113 pISSN: 2005-727X eISSN: 2005-7288

(C) The Korean Society of Medical Education. All rights reserved. This is an open-access article distributed under the terms of the Creative Commons Attribution Non-Commercial License (http:// creativecommons.org/licenses/by-nc/3.0/), which permits unrestricted non-commercial use, distribution, and reproduction in any medium, provided the original work is properly cited. 


\section{서론}

팀바탕학습(team-based learning, TBL)은 문제바탕학습 (problem-based learning, PBL)과 달리 경영학에서 시작된 교육방법이지만, 국내외 의과대학에서 점차 그 활용도가 높 아지고 있는 교육방법 중 하나이다[1]. TBL은 PBL과 마찬가 지로 소그룹 학습을 근간으로 학습자 주도의 적극적 학습 참 여가 가능한 교육방법이면서[2], PBL과 달리 대규모 강의실 에서 개별 교수가 소그룹 학습을 이끌어 갈 수 있다는 점에서 $\mathrm{PBL}$ 에 비해 큰 장점이 있다.

의학교육에서 TBL을 주제로 한 기존 연구는 주로 특정 과 목에서 TBL을 운영한 후 학생 들의 TBL에 대한 만족도나 인 식도를 조사하거나[3,4,5], 학업성취도의 변화[3,5,6,7]를 알 아보는 것에 초점을 맞추었다. 이러한 연구 결과에서 TBL은 팀워크를 강화하고[4], 학습 내용의 이해와 장기 기억에 도움 이 되며[8], 문제해결능력과 임상추론능력을 향상시키며[4], 전문직업성 개발[3]에 유용한 교육방법으로 보고되고 있다. 또한 TBL은 전통적인 교육방법에 비해 학생들의 학업성취도 를 향상시키며[5,7], 이는 의사국가시험에서도 의미 있는 좋 은 결과가 있는 것으로 보고되고 있다[7].

그러나 모든 교육 방법에 장단점이 있듯이 모든 학습자들 이 TBL을 긍정적으로 평가하며, 그 과정에서 긍정적인 학습 경험을 하는 것은 아니다[9]. TBL을 긍정적인 학습경험으로 인식하고 효과적이고 우수한 수행을 보이는 팀도 있지만[10], 그렇지 않은 팀들도 존재한다[11]. TBL을 긍정적인 학습경험 으로 인식하는 데에는 TBL을 위한 교육환경과 지원체계가 잘 갖춰졌는지와 같은 외적인 요인도 중요할 수 있지만[3], 팀 을 구성하고 있는 학생 개인의 특성도 관련이 있다[6]. 개인 특성은 연령, 성별 등과 같은 인구통계학적 특성과 성격, 가 치, 태도와 같은 정의적 특성, 지능, 학업 성적과 같은 인지적 특성이 대표적이다. 구성원들 각자가 지닌 이러한 특성들의 차이에 따라 TBL의 효과를 인식하는 정도는 다르게 나타날 수 있으며[6,12], 이는 곧 TBL의 성패에도 영향을 미칠 수 있 기 때문에 어떤 특성을 지닌 학습자들끼리 같은 팀 혹은 다른 팀으로 구성할 것인가에 대해 관심을 기울일 필요가 있다.

최근 연구에서 팀 성과나 팀 활동에 영향을 미치는 중요한
결정요인으로 팀효능감이 공론화되고 있다[13]. 팀 효능감은 팀에 주어진 과제를 성공적으로 수행할 수 있을 것이라는 팀 에 대한 믿음을 의미하며[13], 팀에서 자신이 무슨 일을 할지 를 선택하고 팀을 위해 얼마만큼의 노력을 기울일 것인지와 관련이 있다. 따라서 팀효능감을 어떻게 지각하느냐에 따라 $\mathrm{TBL}$ 경험을 긍정적 또는 부정적으로 인식할 것이다.

또한 TBL은 팀 활동을 기본으로 하기 때문에 팀 활동 과정 을 구성하는 요인인 대인관계 이해도와 팀 과제 해결을 위한 적극적 태도가 TBL을 긍정적인 학습경험으로 인식하는 데 영향을 줄 수 있다[14]. 대인관계 이해도는 팀원들 간의 관계 형성 측면으로 팀원들이 서로 신뢰하고 이해하는 정도를 말 하며[14], 팀원들이 서로의 강점과 약점 등을 잘 알고 팀원의 행동을 예측하고 상호 요구하는 부분을 보완해 주는 것은 팀 활동에서 중요한 요소 중 하나이다. 팀 과제 해결의 적극성은 팀이 학습해야 할 과제가 무엇인지를 인지하고 해결하기 위 해 보이는 개인의 태도를 의미하는데, 팀 과제 해결에 개인이 어느 정도의 적극성을 갖고 있느냐에 따라 TBL에 대한 인식 은 달라질 것이다.

정의적 특성 이외에도 학업능력과 같은 지적 수준에 따라 $\mathrm{TBL}$ 을 인식하는 정도에 차이가 있다. 간호대학생의 경우 학 업능력에 따라 TBL 인식에 차이가 없었으며[10], 의과대학생 이나 치과대학생의 경우 학업능력이 높은 학생들이 낮은 학 생들에 비해 TBL에 대해 긍정적으로 평가하거나[7], 학업능 력 상위 집단은 팀바탕학습에 대한 만족도가 높다고 보고한 바 있다[6]. 하지만 보건의료계열이라는 동일 학문분야에서 도 연구 결과에 차이가 있어 일반화된 결과를 도출하기에는 연구가 부족한 실정이다.

이와 같이 한 개인이 경험에 대해 인식하는 관점에는 다양 한 개인적 특성이 관련될 수 있는데, TBL 경험에서 이러한 개인적 특성을 고려한 연구는 부족한 편이다. TBL과 같이 팀 으로 학습해야 하는 상황에서 개인적 특성들을 고려하여 팀 원들을 구성한다면 팀이 가진 시너지 효과가 달라질 수 있고, 팀 구성을 어떻게 하느냐에 따라 학습활동이나 학습효과에 있어 분명한 차이가 나타날 수 있다[12]. 따라서 본 연구에서 는 학생 개인이 지닌 특성에 따라 TBL의 경험이나 효과를 어 떻게 인식하고 있으며, 그 차이가 있는지를 분석하고자 하였 다. 즉, 개인의 인구통계학적 특성인 성별, 정의적 특성인 팀 
효능감, 대인관계 이해도, 문제해결에서의 적극성, 인지적 특 성인 학업 능력에 따라 TBL 경험이나 효과를 어떻게 인식하 고 있는지를 살펴보고, TBL 경험을 인식하는 데 영향을 미치 는 요인을 알아보고자 하였다.

\section{대상 및 방법}

\section{1. 대상}

본 연구는 인천광역시에 소재한 의학전문대학원 2학년 심장 학 통합교육과정 중 TBL에 참여한 학생 38명을 연구 대상으로 하였다. 학생의 분포는 남학생이 28 명, 여학생이 10 명이었다.

\section{2. 연구 도구}

\section{1) TBL에 대한 인식도 조사}

학생들의 TBL에 대한 인식도를 조사하기 위해 관련 문헌 고찰을 토대로 설문지를 구성하였다. 그리고 이 측정도구의 타당성을 검증하기 위해 실시한 요인 분석 결과에 따라 3 개 요인으로 구성된 총 12 개 문항을 구성하였다. TBL 인식도는 팀 스킬 향상(I, 5문항), 학습능력 향상(II, 4문항), 팀 학습 향 상(III, 3 문항)의 3 개 요인으로 구성되었으며 전체 변량의 $66.01 \%$ 를 설명하였다. 각 요인이 측정하는 바는 다음과 같다.
팀 스킬 향상 요인은 TBL을 통해 협동능력, 타인의 의견을 존중하는 능력 등과 같은 팀 스킬 향상에 도움이 되었는지를 측정하는 문항들로 구성되었다(예, TBL이 다른 사람의 의견 을 존중하는 능력을 기르는 데 도움이 되었다). 학습능력 향 상 요인은 TBL이 수업내용을 이해하고, 시험문제를 해결하 는 데 도움이 되었는지를 측정하는 문항들로 구성되었다(예, TBL로 배운 내용이 심장학 시험문제를 푸는 데 도움이 되었 다). 마지막으로 팀 학습 향상 요인은 팀으로 학습하는 것이 개별 학습보다 도움이 되었는지, 팀이 함께 문제를 해결하면 서 자신이 잘못 이해하고 있는 부분을 이해하게 되었는지 등 팀으로 학습할 때의 장점과 효과에 대한 인식을 측정하는 하 는 문항들로 구성되었다(예, 혼자 학습하는 것보다 팀으로 학 습하면서 더 많은 것을 배울 수 있었다). 각 요인별 문항의 신 뢰도 값(Cronbach's $\alpha$ 계수)은 팀 스킬 향상 요인이 0.834, 학습능력 향상 요인이 0.773 , 팀 학습 향상 요인이 0.724 이었 다(Table 1). 각 문항에 대한 반응 양식은 Likert식 5점 척도 를 사용하였으며, 점수가 높을수록 각 요인에 대해 긍정적으 로 인식하는 것으로 해석하였다.

\section{2) 학업능력}

학업능력이란 학과 공부를 성공적으로 수행할 수 있는 어휘 적, 수리적 능력 및 논리적 사고력과 노력의 지속성 등을 총괄하 는 학문적 경쟁력(academic competitiveness)을 말한다. 가장 객관적인 학업능력은 흔히 학교 성적(grade point average,

Table 1. Factor Loadings for Team-Based Learning Perception Items

\begin{tabular}{ccccc}
\hline Item & I & II & III & Cronbach's $\alpha$ \\
\hline 12 & 0.813 & -0.477 & 0.322 & \\
8 & 0.798 & -0.429 & 0.081 & \\
9 & 0.790 & -0.383 & 0.533 & 0.834 \\
11 & 0.728 & -0.363 & 0.539 & \\
10 & 0.704 & -0.303 & 0.044 & \\
5 & 0.361 & -0.857 & -0.160 & \\
7 & 0.418 & -0.852 & 0.198 & \\
6 & 0.405 & -0.840 & 0.270 & \\
1 & 0.370 & -0.407 & 0.389 & \\
3 & 0.169 & -0.095 & 0.927 & \\
2 & 0.617 & -0.203 & 0.707 & \\
4 & 0.402 & -0.534 & 0.583 & \\
Percent of variance accounted for & 42.151 & 4.541 & 9.320 & \\
\hline
\end{tabular}

Factor I: Team skill improvement, Factor II: Learning ability improvement, Factor III: Team learning improvement. 
Kwi Hwa Park, et al : Students' Individual Characteristics and the Perception of TBL

GPA)으로 나타난다. 따라서 본 연구에서는 연구 대상 학생들 의 직전 학기까지의 $\mathrm{GPA}$ 를 학업능력으로 정의하였다.

\section{3) 팀 효능감}

학생들의 팀 효능감은 Marshall [15]의 연구에서 사용된 8문 항을 Kwon [14]이 번안한 것을 사용하여 측정하였다. 팀 효능 감을 측정하는 설문 문항으로는 "나는 우리 팀이 팀 과제와 팀 시험을 훌륭하게 해낼 수 있다고 자신한다," "나는 우리 팀이 팀 안에서 활동하는 방법에 관한 지식을 갖고 있다고 확신한다" 등으로 구성되어 있다. 팀 효능감의 신뢰도는 Cronbach's $\alpha$ $=0.900$ 이었다. 각 문항에 대한 반응 양식은 Likert식 5 점 척도 를 사용하였으며, 점수가 높을수록 팀 효능감이 높은 것으로 해석하였다.

\section{4) 대인관계의 이해}

대인관계 이해는 Marshall [15]의 연구에서 사용된 대인관 계의 이해 11 문항을 Kwon [14]이 번안한 것을 사용하여 측 정하였다. 대인관계의 이해를 측정하는 설문 문항으로는 "나 는 다양한 배경을 가진 팀원들 모두와 팀 활동을 잘한다"와 "나는 우리 팀원들의 생각과 의견을 존중한다" 등으로 구성되 어 있다. 대인관계 이해의 신뢰도 계수는 Cronbach's $\alpha=$ 0.869 이다. 각 문항에 대한 반응 양식은 Likert식 5점 척도를 사용하였으며, 점수가 높을수록 대인관계 이해도가 높은 것 으로 해석하였다.

\section{5) 문제해결에서의 적극성}

문제해결에서의 적극성은 Marshall [15]의 연구에서 사용된 문제해결에서의 적극성 8문항을 Kwon [14]이 번안한 것을 사용 하여 측정하였다. 문제해결에서의 적극성을 측정하는 설문 문항 으로는 "나는 과제를 해결하기 위한 해결책을 적극적으로 제시 한다"와 “나는 팀 과제 수행 중에 문제가 발생하면 적극적으로 대안을 찾으려고 한다" 등으로 구성되어 있다. 문제해결에서의 적극성 신뢰도 계수는 Cronbach's $\alpha=0.789$ 이다. 각 문항에 대한 반응 양식은 Likert식 5점 척도를 사용하였으며, 점수가 높을수록 문제해결에서의 적극성이 높은 것으로 해석하였다.

\section{3. 자료 수집}

본 연구는 2012년 5월 14일부터 6월 22일까지 6주가 진행된 심장학 통합교육과정에서 강의시간 130 시간 중 24 시간을 TBL 방식으로 운영한 후 학생들에게 조사를 실시하였다. TBL은
3 단계로 진행하였다. 1 단계는 학생들이 수업에 참여하기 전에 익혀야 할 학습 목표와 사전읽기 자료를 온라인 수업 지원 시스 템을 이용하여 일주일 전에 제시하여 학습해 오게 하였다. 2 단계 는 사전 과제를 잘 수행했는지를 확인하기 위해 개인 학습 준비 도 점검을 위한 시험(individual readiness assurance test, IRAT)을 실시하였고, 동일한 문항을 해결하기 위해 다시 소집 단을 구성하여 토론하고 학습한 후 그룹 시험(group readiness assurance test, GRAT)을 실시한 후 전체 그룹이 토론을 하도록 하였다. 마지막 3 단계는 1,2 단계를 통해 학습한 지식을 바탕으 로 적용하는 단계로, 그룹별로 토의를 통하여 문항을 해결하도 록 하였다. 이와 같이 TBL을 진행하고 연구자가 학생들에게 연구 목적에 대해 설명하고 지시사항을 숙지시킨 후 학생들의 팀 효능감, 대인관계 이해도 문제해결의 적극성과 TBL의 인식 도를 평가하는 설문지를 작성하도록 하였다. 설문 작성에 시간 제한을 두지는 않았으며, 설문지를 완성하는 데 소요되는 시간 은 약 15 분 정도였다. 설문지 작성이 완료된 후에는 연구자가 직접 설문지를 회수하였다.

\section{4. 자료 분석 방법}

본 연구에서 사용된 자료 분석 방법은 다음과 같다. 첫째, 성별에 따른 TBL 인식도의 차이를 살펴보기 위해 $t$-test를 실시하였다. 둘째, 학생들의 학업능력, 팀 효능감, 대인관계의 이해, 문제해결에서의 적극성에 대한 집단별 TBL 인식도 차 이를 살펴보기 위해 analysis of variance를 실시하였다. 각 변인을 상위집단, 중위집단, 하위집단의 3집단으로 구분하기 위해 점수 분포에 따라 상위집단은 상위 $33 \%$, 하위집단은 하 위 $33 \%$ 로 하였고, 나머지 집단은 중위집단으로 구분하였다. 셋째, 학생들의 학업능력, 팀 효능감, 대인관계의 이해, 문제 해결에서의 적극성이 TBL 인식도에 미치는 영향을 분석하기 위해 상관분석(Pearson's r)과 중다회귀분석(stepwise multiple regression analysis)을 수행하였다.

\section{결과}

\section{1. 성별에 따른 $\mathrm{TBL}$ 인식도 차이}

학생들의 성별에 따른 TBL 인식도에 차이가 있는지를 살 
펴보았다. 그 결과 $\mathrm{TBL}$ 인식도 하위요인 중 학습능력 향상 요인에서 성별에 따른 통계적으로 의미 있는 차이가 나타났 으나 $(\mathrm{p}<0.05)$, 팀 스킬 향상 $(\mathrm{p}>0.05)$, 팀 학습 효과 $(\mathrm{p}>0.05)$, $\mathrm{TBL}$ 인식도 전체 $((\mathrm{p}>0.05)$ 에서는 차이가 나타나지 않았다. 즉, 남학생이 여학생보다 TBL이 학습능력 향상에 효과가 있 었다고 인식하는 것으로 나타났다(Table 2).

\section{2. 학업능력에 따른 TBL 인식도 차이}

학생들의 학업능력에 따라 TBL 인식도에 차이가 있는지를 알아보기 위해 학업능력 상위집단, 중위집단, 하위집단의 세 집단으로 구분하여 살펴보았다. 그 결과, $\mathrm{TBL}$ 인식도 하위요 인 중 학습능력 향상요인이 학업능력 수준에 따라 통계적으 로 의미 있는 차이가 나타났으나 $(\mathrm{p}<0.05)$, 팀 스킬 향상 $(\mathrm{p}>0.05)$ 과 팀 학습 요인 $(\mathrm{p}>0.05)$ 에서는 차이가 나타나지
않았다. 또한 $\mathrm{TBL}$ 인식도 전체에서도 의미 있는 차이가 나타 나지 않았다 $(\mathrm{p}>0.05)$. 즉, 학업능력 상위집단의 학생들이 하 위집단 학생들 보다 TBL이 학습능력 향상에 도움이 되었다 고 인식하는 것으로 나타났다(Table 3).

\section{3. 팀 효능감 수준에 따른 TBL 인식도 차이}

학생들의 팀 효능감 수준에 따라 TBL 인식도에 차이가 있 는지를 알아보기 위해 팀 효능감 수준을 상위집단, 중위집단, 하위집단의 세 집단으로 구분하여 살펴보았다. 그 결과 $\mathrm{TBL}$ 인식도 하위요인 중 팀 스킬 향상 요인과 팀 학습 효과 요인이 팀 효능감 수준에 따라 유의미한 차이가 나타났으며, TBL 인 식도 전체에서도 의미 있는 차이가 나타났다 $(\mathrm{p}<0.05)$. 그러 나 학습능력 향상 요인에서는 차이가 나타나지 않았다 $(\mathrm{p}>$ 0.05). 즉, 팀 효능감 상위집단의 학생들이 하위집단 학생들

Table 2. Gender Differences in Perception of Team-Based Learning

\begin{tabular}{llllccc}
\hline \multicolumn{1}{c}{ Variable } & Gender & No. & Mean & SD & t & p-value \\
\hline Team skill & Male & 28 & 4.09 & 0.58 & 0.814 & 0.421 \\
\multirow{2}{*}{ Learning ability } & Female & 10 & 3.92 & 0.58 & & \\
& Male & 28 & 4.05 & 0.47 & 2.245 & 0.031 \\
\multirow{2}{*}{ Team learning } & Female & 10 & 3.65 & 0.54 & & \\
\multirow{3}{*}{ Total of TBL perception } & Male & 28 & 4.08 & 0.57 & 0.247 & 0.807 \\
& Female & 10 & 4.03 & 0.48 & & \multirow{2}{*}{0.203} \\
& Male & 28 & 4.08 & 0.45 & 1.297 & \\
\hline
\end{tabular}

TBL: Team-based learning, SD: Standard deviation.

Table 3. Differences in Perception of Team-Based Learning by Academic Ability

\begin{tabular}{|c|c|c|c|c|c|c|c|}
\hline Variable & Group & No. & Mean & SD & $\mathrm{F}$ & $p$-value & Scheffé \\
\hline \multirow[t]{3}{*}{ Team skill } & High & 12 & 4.07 & 0.63 & 1.199 & 0.314 & \\
\hline & Middle & 16 & 4.18 & 0.61 & & & \\
\hline & Low & 10 & 3.82 & 0.42 & & & \\
\hline \multirow[t]{3}{*}{ Learning ability } & High $^{\text {al }}$ & 12 & 4.31 & 0.39 & 7.236 & 0.002 & $a|>c|^{*}$ \\
\hline & Middle $^{\mathrm{bl}}$ & 16 & 3.89 & 0.50 & & & \\
\hline & $\operatorname{Low}^{\mathrm{cl}}$ & 10 & 3.60 & 0.41 & & & \\
\hline \multirow[t]{3}{*}{ Team learning } & High & 12 & 3.97 & 0.63 & 1.211 & 0.310 & \\
\hline & Middle & 16 & 4.23 & 0.47 & & & \\
\hline & Low & 10 & 3.93 & 0.54 & & & \\
\hline \multirow[t]{3}{*}{ Total of TBL perception } & High & 12 & 4.12 & 0.50 & 2.086 & 0.139 & \\
\hline & Middle & 16 & 4.10 & 0.40 & & & \\
\hline & Low & 10 & 3.78 & 0.38 & & & \\
\hline
\end{tabular}

TBL: Team-based learning, SD: Standard deviation. ${ }^{*} p<0.05$. 
보다 TBL이 팀 스킬 향상에 도움이 되었고, 팀으로 학습하는 것이 효과가 있다고 인식하는 것으로 나타났다(Table 4).

\section{4. 대인관계 이해도 수준에 따른 TBL 인식도 차이}

학생들의 대인관계 이해도 수준에 따라 TBL 인식도에 차이 가 있는지를 알아보기 위해 대인관계 지각 수준에 따라 상위집 단, 중위집단, 하위집단의 세 집단으로 구분하여 살펴보았다. 그 결과 $\mathrm{TBL}$ 인식도 하위요인 중 팀 스킬 향상 요인이 대인관 계 이해도 수준에 따라 유의미한 차이가 나타났으며, TBL 인 식도 전체에서도 의미 있는 차이가 나타났다 $(\mathrm{p}<0.05)$. 그러 나 학습능력 향상 요인과 팀 학습 효과 요인은 유의미한 차이
가 나타나지 않았다 $(\mathrm{p}>0.05)$. 즉, 대인관계 이해도 상위집단 의 학생들이 하위집단 학생들보다 TBL이 팀 스킬 향상에 도움 이 된다고 인식하는 것으로 나타났다(Table 5).

\section{5. 문제해결의 적극성 수준에 따른 TBL 인식도 차이}

학생들의 문제해결의 적극성 수준에 따라 $\mathrm{TBL}$ 인식도에 차 이가 있는지를 알아보기 위해 문제해결의 적극성 수준에 따라 상위집단, 중위집단, 하위집단의 세 집단으로 구분하여 살펴보 았다. 그 결과 $\mathrm{TBL}$ 인식도 하위요인 중 팀 스킬 향상 요인이 문제해결의 적극성 수준에 따른 유의미한 차이가 나타났으며, $\mathrm{TBL}$ 인식도 전체에서도 의미있는 차이가 나타났다 $(\mathrm{p}<0.05)$.

Table 4. Differences in Perception of Team-Based Learning by Level of Team Efficacy

\begin{tabular}{|c|c|c|c|c|c|c|c|}
\hline Variable & Group & No. & Mean & SD & $\mathrm{F}$ & $\mathrm{p}$-value & Scheffé \\
\hline \multirow[t]{3}{*}{ Team skill } & $\operatorname{High}^{\mathrm{al}}$ & 11 & 4.38 & 0.64 & 4.094 & 0.025 & a) $>\left.c\right|^{*}$ \\
\hline & Middle ${ }^{b l}$ & 17 & 4.02 & 0.36 & & & \\
\hline & Low $^{c \mid}$ & 10 & 3.72 & 0.64 & & & \\
\hline \multirow[t]{3}{*}{ Learning ability } & High & 11 & 4.00 & 0.54 & 2.091 & 0.139 & \\
\hline & Middle & 17 & 4.07 & 0.47 & & & \\
\hline & Low & 10 & 3.68 & 0.50 & & & \\
\hline \multirow[t]{3}{*}{ Team learning } & $\operatorname{High}^{\text {al }}$ & 11 & 4.42 & 0.62 & 5.892 & 0.006 & a) $>\left.c\right|^{*}$ \\
\hline & Middle $^{b /}$ & 17 & 4.06 & 0.44 & & & \\
\hline & Low $^{c \mid}$ & 10 & 3.70 & 0.37 & & & \\
\hline \multirow[t]{3}{*}{ Total of TBL perception } & $\operatorname{High}^{\text {al }}$ & 11 & 4.27 & 0.50 & 5.551 & 0.008 & a) $>\left.c\right|^{*}$ \\
\hline & Middle $^{\text {bl }}$ & 17 & 4.05 & 0.26 & & & \\
\hline & Low $^{c l}$ & 10 & 3.70 & 0.45 & & & \\
\hline
\end{tabular}

TBL: Team-based learning, SD: Standard deviation. ${ }^{*} p<0.05$.

Table 5. Differences in Perception of Team-Based Learning by Level of Interpersonal Understanding

\begin{tabular}{|c|c|c|c|c|c|c|c|}
\hline Variable & Group & No. & Mean & SD & $\mathrm{F}$ & $p$-value & Scheffé \\
\hline \multirow[t]{3}{*}{ Team skill } & High $^{\text {al }}$ & 14 & 4.41 & 0.56 & 6.854 & 0.003 & a) $>\left.c\right|^{*}$ \\
\hline & Middle $^{\text {bl }}$ & 11 & 3.98 & 0.46 & & & \\
\hline & Low $^{\mathrm{cl}}$ & 13 & 3.71 & 0.47 & & & \\
\hline \multirow[t]{3}{*}{ Learning ability } & High & 14 & 4.07 & 0.54 & 1.051 & 0.360 & \\
\hline & Middle & 11 & 3.98 & 0.49 & & & \\
\hline & Low & 13 & 3.79 & 0.50 & & & \\
\hline \multirow[t]{3}{*}{ Team learning } & High & 14 & 4.21 & 0.59 & 2.237 & 0.122 & \\
\hline & Middle & 11 & 4.18 & 0.38 & & & \\
\hline & Low & 13 & 3.82 & 0.55 & & & \\
\hline \multirow[t]{3}{*}{ Total of TBL perception } & $\mathrm{High}^{\mathrm{al}}$ & 14 & 4.23 & 0.44 & 4.392 & 0.020 & $a|>c|^{*}$ \\
\hline & Middle $^{\mathrm{bl}}$ & 11 & 4.05 & 0.39 & & & \\
\hline & Low $^{c l}$ & 13 & 3.77 & 0.37 & & & \\
\hline
\end{tabular}

TBL: Team-based learning, SD: Standard deviation. ${ }^{*} p<0.05$. 
그러나 학습능력 향상 요인과 팀 학습 효과 요인에서는 차이가 나타나지 않았다 $(\mathrm{p}>0.05)$. 즉, 문제해결의 적극성 상위집단의 학생들이 중위집단이나 하위집단 학생들 보다 TBL이 팀 스킬 향상에 도움이 된다고 인식하는 것으로 나타났다(Table 6).

\section{TBL 인식도에 영향을 미치는 변인}

학생들의 학업능력, 팀 효능감, 대인관계의 이해도, 문제해 결의 적극성, $\mathrm{TBL}$ 인식도의 평균 및 표준편차, 그리고 각 변 인들 간의 상호상관을 살펴보았다. 학생들의 TBL 인식도는 팀 효능감 $(r=0.456, p<0.01)$, 대인관계 $(r=0.417, p<0.01)$, 문 제해결의 적극성 $(\mathrm{r}=0.471, \mathrm{p}<0.01)$ 과 유의한 정적 상관이 있 는 것으로 나타났으며, 학업능력 $(\mathrm{r}=0.255, \mathrm{p}>0.05)$ 과는 유의 한 상관관계를 보이지 않았다. 학업능력은 문제해결의 적극 성 $(\mathrm{r}=0.362, \mathrm{p}<0.01)$ 과는 유의한 정적 상관이 있는 것으로 나타났으며, 팀 효능감 $(\mathrm{r}=0.151, \mathrm{p}>0.05)$, 대인관계 이해( $\mathrm{r}=$
0.199, $\mathrm{p}>0.05$ )와는 유의한 상관관계를 보이지 않았다. 팀 효 능감은 대인관계 이해 $(\mathrm{r}=0.538, \mathrm{p}<0.01)$ 와는 유의한 정적 상 관이 있는 것으로 나타났으며, 문제해결의 적극성 $(\mathrm{r}=0.319$, $\mathrm{p}>0.05)$ 과는 유의한 상관관계를 보이지 않았다. 대인관계는 문제해결의 적극성 $(\mathrm{r}=0.539, \mathrm{p}<0.01)$ 과는 유의한 정적 상관 이 있는 것으로 나타났다(Table 7).

TBL 인식도와 유의한 상관을 보이는 변인들이 TBL 인식 도를 얼마나 설명해 주는지를 알아보기 위해 TBL 인식도를 종속변인으로 학업능력, 팀 효능감, 대인관계, 문제해결의 적 극성을 독립변인으로 한 단계적 다중회귀분석을 수행하였다. 문제해결의 적극성 $(\beta=0.362)$, 팀 효능감 $(\beta=0.340)$ 이 TBL 인식도를 설명해 주는 변인으로 나타났으며, TBL 인식도의 전체 변량 중 $32.5 \%$ 를 설명하는 것으로 나타났다(Table 8). 즉, 문제해결에 적극성을 보이며, 팀 효능감이 높을수록 TBL 을 긍정적으로 지각함을 알 수 있다.

Table 6. Differences in Perception of Team-Based Learning by Level of Proactivity in Problem-Solving

\begin{tabular}{|c|c|c|c|c|c|c|c|}
\hline Variable & Group & No. & Mean & SD & $\mathrm{F}$ & $p$-value & Scheffé \\
\hline \multirow[t]{3}{*}{ Team skill } & High $^{\text {al }}$ & 14 & 4.47 & 0.42 & 8.584 & 0.001 & $a|>c|^{*}$ \\
\hline & Middle $^{b /}$ & 11 & 3.84 & 0.58 & & & $a|>b|^{*}$ \\
\hline & Low $^{c l}$ & 13 & 3.77 & 0.46 & & & \\
\hline \multirow[t]{3}{*}{ Learning ability } & High & 14 & 4.11 & 0.51 & 2.707 & 0.081 & \\
\hline & Middle & 11 & 4.05 & 0.46 & & & \\
\hline & Low & 13 & 3.69 & 0.50 & & & \\
\hline \multirow[t]{3}{*}{ Team learning } & High & 14 & 4.19 & 0.58 & 0.657 & 0.525 & \\
\hline & Middle & 11 & 3.94 & 0.49 & & & \\
\hline & Low & 13 & 4.05 & 0.56 & & & \\
\hline \multirow[t]{3}{*}{ Total of TBL perception } & $\operatorname{High}^{\mathrm{al}}$ & 14 & 4.26 & 0.38 & 3.803 & 0.032 & a) $>\left.c\right|^{*}$ \\
\hline & Middle $^{\mathrm{bl}}$ & 11 & 3.94 & 0.45 & & & \\
\hline & $\operatorname{Low}^{c l}$ & 13 & 3.84 & 0.41 & & & \\
\hline
\end{tabular}

TBL: Team-based learning, SD: Standard deviation.

${ }^{*} p<0.05$.

Table 7. Means, Standard Deviations, and Correlation among Variables

\begin{tabular}{lccccccc}
\hline & Mean & SD & 1 & 2 & 3 & 4 & 5 \\
\hline 1. Perception of TBL & 4.02 & 0.44 & - & & & & \\
2. Academic ability & 3.30 & 0.41 & 0.26 & - & & & \\
3. Team efficacy & 4.14 & 0.54 & $0.46^{* *}$ & 0.15 & - & & \\
4. Interpersonal understanding & 4.22 & 0.45 & $0.42^{* *}$ & 0.20 & $0.54^{* *}$ & - \\
5. Proactivity in problem-solving & 4.03 & 0.50 & $0.47^{* *}$ & $0.36^{*}$ & 0.32 & $0.54^{* *}$ & - \\
\hline
\end{tabular}

SD: Standard deviation, TBL: Team-based learning. ${ }^{*} p<0.05,{ }^{*} p<0.01$. 
Kwi Hwa Park, et al : Students' Individual Characteristics and the Perception of TBL

Table 8. Stepwise Multiple Regression Analysis of Predicting Perception of Team-Based Learning

\begin{tabular}{clccccc}
\hline Dependent & \multicolumn{1}{c}{ Predictors } & B & SE B & $\beta$ & $\Delta R^{2}$ & $R^{2}$ \\
\hline Perception of TBL effect & (Constant) & 1.599 & 0.593 & & & \\
& Proactivity in problem-solving & 0.319 & 0.129 & $0.362^{*}$ & 0.222 & 0.325 \\
& Team efficacy & 0.276 & 0.119 & $0.340^{*}$ & 0.103 & \\
\hline
\end{tabular}

TBL: Team-based learning.

${ }^{*} \mathrm{p}<0.05$.

\section{고찰}

본 연구에서는 학생의 개인적 특성에 따라 $\mathrm{TBL}$ 효과를 어 떻게 인식하고 있으며, 그 차이가 있는지를 분석하여 TBL에 서 팀을 구성하고 TBL의 효과를 증진시키는 데 필요한 기초 자료를 제공하고자 하였다.

TBL은 학생들에게 흥미와 동기유발을 주는 긍정적인 학습 활동이며, 의학교육에서도 활용 가능한 교육방법이며, 시간과 노력을 들인 만큼 가치 있는 경험으로 평가되고 있다[3]. 그러 나 TBL은 팀을 통한 학습이기 때문에 팀 구성원 개인이 지닌 특성의 다양성으로 인해 $\mathrm{TBL}$ 경험을 인식하는 데에도 차이가 있으며, 궁극적으로는 이것이 팀 구성원의 능동적 상호작용과 팀 성과를 결정하는 데에도 영향을 미친다. 즉, 구성원들 각자 가 가진 특성들의 차이에 따라 팀 다양성의 영향력은 다르게 나타날 수 있으며 이는 곧 성과에 영향을 미치기 때문이다.

인구학적 특성 중 성별에서는 남학생이 여학생보다 TBL이 학습능력을 향상시키는 데 도움이 되었다고 인식하였다. 남 학생이 많은 학급에서 협동학습을 실시하였을 때가 여성이 많은 학급에서 실시하였을 때보다 학습태도에서 효과적이라 는 보고가 있는데, 이는 남학생이 여학생에 비해 수업에 있어 서 다소 산만하지만 능동적이고 적극적으로 참여하는 특성이 있기 때문이다[16]. 본 연구에서도 남학생 수가 많은 학습 환 경으로 인해 능동적인 참여가 이뤄졌고 이는 학생들의 $\mathrm{TBL}$ 효과 인식과 긍정적인 관련성이 있었다고 할 수 있다. 그러나 Wiener et al. [5]의 연구에 의하면, 여학생이 남학생보다 $\mathrm{TBL}$ 식 접근방식에 대해 만족한다는 응답이 높았다는 연구 결과와는 상반된다.

학업능력 상위집단의 학생들이 하위집단 학생들보다 TBL 이 학습능력 향상에 도움이 되었다고 인식하였다. 이는 학업
능력이 높은 학생들이 낮은 학생들에 비해 TBL에 대해 긍정 적으로 평가하는 경향이 있다고 보고한 연구 결과와 일치한 다[7]. 또한 한 연구에 의하면 학업능력에 따른 수업만족도에 서 $\mathrm{TBL}$ 은 성취도 상위집단에서, 강의방법은 하위집단에서 만족도가 높았다고 보고하였는데[8], 이는 본 연구 결과를 지 지해 주는 결과이다. 반면, 간호대학생을 대상으로 한 Roh et al. [10]의 연구에서는 학업능력 수준에 따라 TBL 인식도에 차이가 없었다고 하여 본 연구 결과와는 상반된다. 학업능력 이 높은 학생들은 자기주도적이고 적극적으로 학습에 참여하 는 경향이 있는데[17], TBL은 자기주도적으로 사전 과제를 학습하고 능동적으로 학습에 참여하여 팀원들을 설득하는 것 이 요구되는 수업방식이기 때문에 학업능력이 높은 학생들의 특성과 부합되는 부분이 있어 긍정적으로 평가를 했을 것이 다. 또한 학업능력이 뛰어난 학생들은 변화된 교육방법의 장 점을 이해하고 빠르게 대처하는 능력이 있어 TBL으로의 변 화도 쉽게 받아들였다고 볼 수 있다[7]. 이처럼 팀을 구성할 때 학업능력은 중요한 특성 중 하나인데, 학업능력에 따라 팀 을 이질적으로 구성하면, 능력이 낮은 학습자들은 학업 성취 면에서 자신보다 능력이 높은 학습자들로부터 도움을 얻을 수 있는 장점이 있지만, 반면에 능력이 뛰어난 학습자들은 오 히려 학업성취에 방해를 받을 수 있기 때문에[18], 동질 집단 으로 구성하는 것이 더 바람직할 수도 있다. 이처럼 합의된 결 론은 없지만, 본 연구 결과를 토대로 볼 때 TBL 도입 초기에 는 학업능력에 따라 이질적으로 팀을 구성하여 TBL에서의 상호작용효과를 높여보는 것을 고려해 볼 수 있을 것이며, TBL에 익숙해지고 난 후 팀 구성을 달리하여 그 차이를 분석 해 볼 필요도 있을 것이다.

TBL에서 어떠한 학습자 특성을 고려한 팀 구성이 학습 효 과의 극대화에 영향을 미칠 수 있느냐가 관심이 되는데, 그 요 인 중에 하나가 팀 효능감이다. 본 연구에서는 팀 효능감 상위 
집단의 학생들이 하위집단 학생들 보다 TBL이 팀 스킬 향상 에 도움이 되었고, 팀으로 학습하는 것이 효과가 있다고 인식 하는 것으로 나타났다. 팀 효능감이 높을수록 팀 학습에 적극 참여하게 되고[19], 이는 TBL을 긍정적인 학습경험으로 인식 하는 데 영향을 주었다고 할 수 있다. 따라서 TBL에 대한 긍 정적인 인식도를 높이기 위해서는 팀 효능감의 정도에 따라 팀을 구성하여야 하며, 나아가 학생들이 팀 효능감을 높일 수 있는 방안을 마련하는 것이 필요하다. 효능감을 증진시키는 원동력에는 구체적인 성공경험이나 긍정적인 피드백 제공이 있다. 따라서 TBL 진행 과정에서 팀 활동이나 팀 성과에 대 해 적절한 피드백이나 보상 시스템을 마련하여 팀 효능감을 높임으로써 학생들이 TBL에 보다 긍정적으로 참여할 수 있 도록 유도하여야 한다.

대인관계 이해도와 문제해결의 적극성 상위집단의 학생들 이 하위집단 학생들 보다 TBL이 팀 스킬 향상에 도움이 되며, $\mathrm{TBL}$ 을 긍정적인 학습경험으로 인식하는 것으로 나타났다. 그 중에서도 팀 과제해결을 위한 적극성은 상위, 중위, 하위집 단을 명확히 구분해 주는 특성으로 나타났다. 경영대학원에 서 팀 학습에 영향을 미치는 프로세스 요인에 대한 연구에서 관계지향적 측면에서는 대인관계의 이해도가 과제지향적 측 면에서는 문제해결에서의 적극성이 유의미한 요인이라고 보 고가 있어 본 연구를 뒷받침해 주고 있다[20]. 이는 TBL을 긍 정적인 학습경험으로 인식하기 위해서는 팀원들과의 관계와 공동으로 주어진 과제를 해결하기 위한 팀원 개인의 적극적 인 태도가 관련이 있음을 시사하는 것이다.

회귀분석 결과에서는 문제해결에 적극성을 보이며, 팀 효 능감이 높을수록 $\mathrm{TBL}$ 을 긍정적으로 지각하는 것으로 나타났 다. 즉, 여러 요인 중에서도 이 두 요인이 TBL 인식도를 유의 미하게 설명해 주는 변인으로 나타났다. 팀효능감이 높은 학 생들은 팀을 위해 무엇을 해야 할지 알고 어려운 과제도 도전 하려는 경향이 높기 때문에[19] 팀 학습에 대한 기여도가 높 을 것이다. 또한 팀에 주어진 과제 해결을 위해 적극성을 보이 는 학생들이 많을수록 팀 구성원의 상호 작용은 높아질 것이 며, 이는 팀에 긍정적인 영향을 미치고, TBL을 긍정적으로 인식하는 데에도 영향을 미쳤다고 할 수 있다. 따라서 팀 구성 에 있어 적극성을 보이는 학생들을 팀별로 적절히 배치하는 것이 필요할 것으로 보인다.
이처럼 팀 다양성(team diversity)은 함께 과업을 수행하는 팀 구성원들 사이의 인구학적, 정의적, 인지적 특성들이 이질 적인 정도로 구성원들 각자가 가진 특성들의 차이에 따라 팀 다양성의 영향력은 다르게 나타나며, 이는 곧 성과에 영향을 미치게 된다. 이러한 관점에서 본 연구는 팀 학습에 대해 긍정 적으로 인식하고, 이것이 팀 학습에 긍정적인 효과로 작용하 기 위해서는 팀 학습을 계획할 때 다양한 요소가 고려된 팀 구성을 해야 한다는 점에서 시사하는 바가 크다. 이러한 구성 을 위해서는 성별, 학업능력, 팀 효능감, 대인관계능력, 문제 해결의 적극성과 같은 개인적 특성을 충분히 고려하여 팀 활 동이 긍정적인 시너지 효과가 일어날 수 있도록 해야 할 것이 다. 그리고 교수자가 개인별 특성들을 잘 파악하고 있다면 팀 활동에서 개인의 역할과 참여에 적절한 조언을 해 줄 수도 있 기 때문에 팀 학습의 질을 향상하는 데에도 기여할 것이다.

그러나 본 연구는 일개 의학전문대학원만을 대상으로 하 였기 때문에 추후 의과대학생 및 보다 많은 의학전문대학원 생을 대상으로 추가 연구를 진행하여 그 결과를 일반화 할 필 요가 있으며, 의과대학생과 의학전문대학원생 간 비교 연구 를 통해 차별화된 프로그램을 제공할 수 있는 기초 자료를 마 련하여야 할 것이다. 본 연구 진행과정에서 $\mathrm{TBL}$ 을 시행한 후 개인적 특성과 TBL 인식도를 조사하였다. 이로 인해 개인적 특성보다 TBL 경험 자체가 TBL 인식도에 영향을 미쳤을 가 능성도 있기 때문에 추후 연구과정에서는 이 점을 고려한 연 구 진행이 필요할 것이다. 좀 더 적극적인 토론 활동 유발을 위해서는 성격, 학생들 간의 친밀도 등도 고려한다면 좀 더 탄 력 있는 팀 활동을 기대할 수 있기 때문에[12] 향후에는 이러 한 개인적 특성을 고려한 추가 연구가 진행되어야 할 것이며, 개인적 특성을 고려한 팀 구성과 그렇지 않은 팀 구성에서 $\mathrm{TBL}$ 성취도를 비교 분석하는 연구도 진행될 필요가 있다.

Acknowledgements: None.

Funding: None.

Conflicts of interest: None. 


\section{REFERENCES}

1. Thompson BM, Schneider VF, Haidet P, Levine RE, McMahon KK, Perkowski LC, Richards BF. Team-based learning at ten medical schools: two years later. Med Educ 2007; 41: 250-257.

2. Haidet P, O'Malley KJ, Richards B. An initial experience with "team learning" in medical education. Acad Med 2002; 77: 40-44.

3. Cho AR, Han SI, Yoon SH, Park JH, Yoo NJ, Kim S. Methods of effective team-based learning administration and expected effects on medical education. Korean J Med Educ 2010; 22: 47-55.

4. Parmelee DX, DeStephen D, Borges NJ. Medical students' attitudes about team-based learning in a pre-clinical curriculum. Med Educ Online 2009; 14: 1.

5. Wiener H, Plass H, Marz R. Team-based learning in intensive course format for first-year medical students. Croat Med J 2009; 50: 69-76.

6. Park IS, Kim DK. Effect of TBL (team-based learning) on oral prophylaxis education of dental hygiene curriculum. J Korean Acad Dent Health 2009; 33: 125-133.

7. Vasan NS, DeFouw DO, Compton S. A survey of student perceptions of team-based learning in anatomy curriculum: favorable views unrelated to grades. Anat Sci Educ 2009; 2: 150-155.

8. McInerney MJ, Fink LD. Team-based learning enhances long-term retention and critical thinking in an undergraduate microbial physiology course. Microbiol Educ 2003; 4: 3-12.

9. Hampton DR, Grudnitski G. Does cooperative learning mean equal learning? J Educ Bus 1996; 72: 5-7.

10. Roh YS, Ryoo EN, Choi DW, Baek SS, Kim SS. A survey of student perceptions, academic achievement, and satisfaction of team-based learning in a nursing course. J Korean Acad Soc Nurs Educ 2012; 18: 239-
247.

11. Mennenga HA. Team-based learning: engagement and accountability with psychometric analysis of a new instrument [dissertation]. [Las Vegas, USA]: University of Nevada; 2010.

12. Kang I, Jung ES. A qualitative analysis on the differences between the student-selected teams and the learning style-based teams in terms of their satisfactory levels on the learning activities. Korean J Educ Methodol Stud 2010; 22: 67-95.

13. Lindsley DH, Brass DJ, Thomas JB. Efficacy-performance spirals: a multilevel perspective. Acad Manage Rev 1995; 20: 645-678.

14. Kwon EM. The correlation among team efficacy, interpersonal understanding, proactivity in problem solving and team performance [master's thesis]. [Seoul, Korea]: Ewha Womans University; 2010.

15. Marshall LC. The relationship between efficacy, teamwork, effort and patient satisfaction [dissertation]. [Los Angeles, USA]: University of Southern California; 2003.

16. Park IS. The effect of cooperative learning on academic achievement and attitude: a meta-analysis [master's thesis]. [Cheongju, Korea]: Korea National University of Education; 2005.

17. Cho HS. A study on the critical thinking disposition and self-directed learning, academic achievement of nursing students. J Korean Soc Health Stat 2007; 32: 57-72.

18. Park SI, Leem J. A review for the educational application of computer-assisted cooperative learning. Korean J Educ Res 1991; 29: 157-176.

19. Park MS. A longitudinal study on the factors affecting team performance among college students: the development of team efficacy perspective [master's thesis]. [Cheonan, Korea]: Hoseo University; 2010.

20. Druskat VU, Kayes DC. Learning versus performance in short-term project teams. Small Group Res 2000; 31: 328-353. 